\title{
Synchronizing female rats' reproductive and metabolic hormones through dietary soy isoflavone
}

\author{
M. Nisa ${ }^{1}$, A. Saleem ${ }^{1}$, H. Anwar ${ }^{2}$, M.K. Khan ${ }^{1}$ and A. Masroor \\ ${ }^{1}$ Institute of Home and Food Sciences and \\ ${ }^{2}$ Department of Physiology, Government College University Faisalabad, Pakistan.
}

Soy isoflavones (S) are the phytoestrogens used as estrogenic replacement (ER) therapy by weaker ER-binding to regulate the reproductive hormones ${ }^{(1)}$. The objective of this study was to examine the effect of various levels of S on dry matter (DM) intake, DM digestibly, body weight (BW), lipid profile, insulin and reproductive hormones of healthy young female rats model.

Thirty-Six Wistar 45 days old rats weighing $95 \pm 5 \mathrm{~g}$ were divided into 4 groups, each having 9 rats: C (control: without S), S5 (S $0.05 \mathrm{~g} / \mathrm{Kg} \mathrm{BW}$ ), S10 (S 0.10g/Kg BW) and $\mathbf{S 1 5}$ (S 0.15g/Kg BW). Soy isoflavones were given through the oral gavage ${ }^{(2)}$. Food and water were offered ad libitum and intake was recorded daily. Body weight was recorded on weekly basis. During last week of trial, collected feces by total collection method ${ }^{(3)}$ and blood samples were used to calculate nutrient digestibility and biochemical analysis respectively. The Completely Randomized Design and LSD test were used to analyze the data ${ }^{(4)}$.

Significant decreased $(\mathrm{P}<0.05)$ dry matter intake was observed in rats fed S5, S10 and S15 diets to that of C group (Fig. 1). DM digestibility was significantly $(\mathrm{P}<0.05)$ increased in rats fed $\mathrm{S} 5$ and $\mathrm{C}$ diets. Crude protein, fiber and ether extract intake and digestibility showed similar trend as in DM (data not shown). Non-significant $(\mathrm{P}>0.05)$ change in final BW was noticed in all rats treated with S diets.Fig. 2 shows that cholesterol, triglyceride and low-density lipoprotein (LDL) was lowest in rats fed with S15 diet, whilst high density lipoproteins (HDL) highest increase was observed in rats fed same diet. In rats fed S15, an increase in serum prolactin and estrogen level as well as decrease in follicle stimulating hormone (FSH), progesterone and insulin was noticed (Fig.3; $\mathrm{P}<0.05)$. Non-significant $(\mathrm{P}>0.05)$ change was observed in testosterone and luteinizing hormone (LH).

In conclusion, S15 diet was found to be effective to synchronize the reproductive hormones in female healthy rats. However longterm administration of this level may cause toxicity. So, long term study with $\mathrm{S}$ in healthy young female rats is required for future investigations.

Fig.1: DM intake, DM digestibility and final weight in female rats fed $\mathrm{S}$ and $\mathrm{C}$ diets

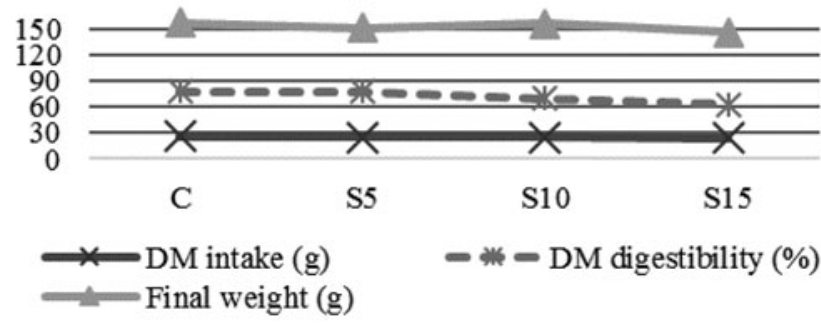

Fig. 3: Hormonal Profile

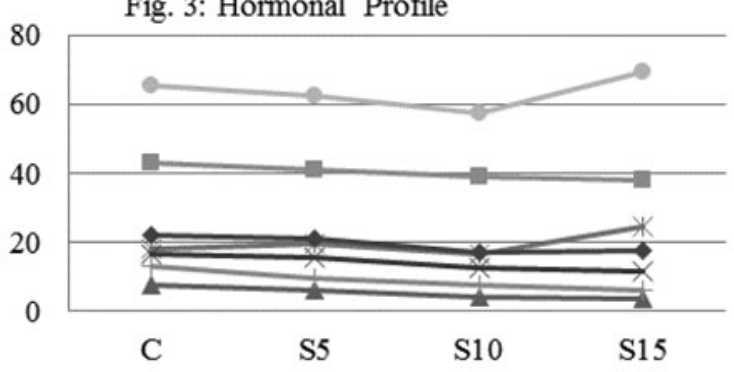

Fig. 2: Lipid Profile

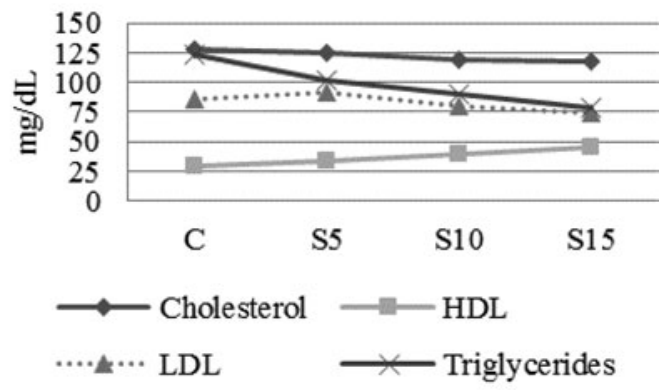

1. Ososki AL \& Edward JK (2003) Phy. Res 17, 845-986.

2. Turner PV (2012) J American Asso Lab Anim Sci 51, 25-30.

3. Nisa M et al. (2008) Asian Aust J Anim Sci 21, 1303-1311.

4. Steel RGD et al. (1997) A Biometrical Approach. 3rd Edition, 352-358. 